\title{
sensors
}

ISSN 1424-8220

(C) 2002 by MDPI

http://www.mdpi.net/sensors

\section{Chemical detection in liquid media with a refractometric sensor based on a multimode optical fibre}

\author{
K. Cherif ${ }^{1}$, S. Hleli ${ }^{1}$, A.Abdelghani ${ }^{1^{*}}$, N.Jaffrezic-Renault ${ }^{2}$ and V. Matejec $^{3}$ \\ ${ }^{1}$ Unité de Recherche de Physique des Semiconducteurs, IPEST, La Marsa, 2070 Tunis, Tunisia. Tel: \\ 0021671740048 . Fax: 0021671746551. \\ 2 IFOS, Ecole Centrale de Lyon, 36 Avenue Guy de Collongue, 69131 Ecully, France \\ ${ }^{3}$ Institute of Radio Engineering and Electronics, Academy of Sciences of the Czech Republic, \\ Chaberska 57, 18251 Prague 8, Czech Republic. \\ * Author to whom correspondence should be addressed. E-mail: aabdelghani@mailcity.com
}

Received: 10 May 2002 / Accepted: 30 May 2002 / Published: 20 June 2002

\begin{abstract}
In this paper the physical basis for the design of an optical fibre sensor suited for aqueous medium and gas phase based on the excitation of an evanescent wave at the core/cladding interface is developed. The detection based on the refractive index changes (between 1.41 and 1.45) of the infinite dielectric medium which can be an electrolyte or a sol-gel polymer deposited on the uncladed part of the fibre. Refractive indices of absorbent and volatile compounds such as fuel and unleaded gas were determined. Using a xerogel sensing layer as optical cladding, toluene detection in water was performed. The observed sensitivity is linear and the detection limit is $1 \%$ (in volume) toluene in water.
\end{abstract}

Key words: Optical fibre, Refractometer, Fuel control, Toluene detection

\section{Introduction}

In the last decade, industry had a lot of effort to develop a method and instrumentation to monitor pollution in river water and in surface water. The role of rivers as a vital source of drinking water as well as for industrial/agricultural processes necessitates continuous control of the water quality. This monitoring should be effected at pumping sites and the various input sites where pollution may occur. In consequence, the quality of major rivers should be monitored continuously and regularly from source to coast. The application of such a control mechanism is that the total number of analytes to be 
monitored in raw water is estimated to increase by approximately $10 \%$ per annum. This places high demands on instrumentation, costs and labour.

Furthermore, current techniques to monitor pollution in river water are, generally, not appropriate. Although such techniques are capable for measuring a large proportion of the analyte spectrum encountered at trace level, these techniques are mostly laboratory-based, expensive and time consuming. There is, therefore, a clear requirement for automated, remote sensing and/or transportable multi-analyte measurement systems. Due to this fact, recently many authors have developed chemical sensors which can be used routinely, with fast response and with similar accuracy to conventional instrumentation. However, the sensors represent cheaper alternatives to conventional analytical instruments.

Several physical phenomena for chemical sensor application have been used. For example, authors use Surface Plasmon Resonance (SPR) on optical fibre [1-4] as a technique for gas and bio-chemical detection. Recently, an increase interest in the application of porous layers prepared by the sol-gel method for the development of fibre-optic chemical sensors have been observed. Employing the solgel technique, fibre-optic sensors for $\mathrm{pH}$ sensing, oxygen sensing or the detection of chlorinated hydrocarbons can be obtained [5-7].

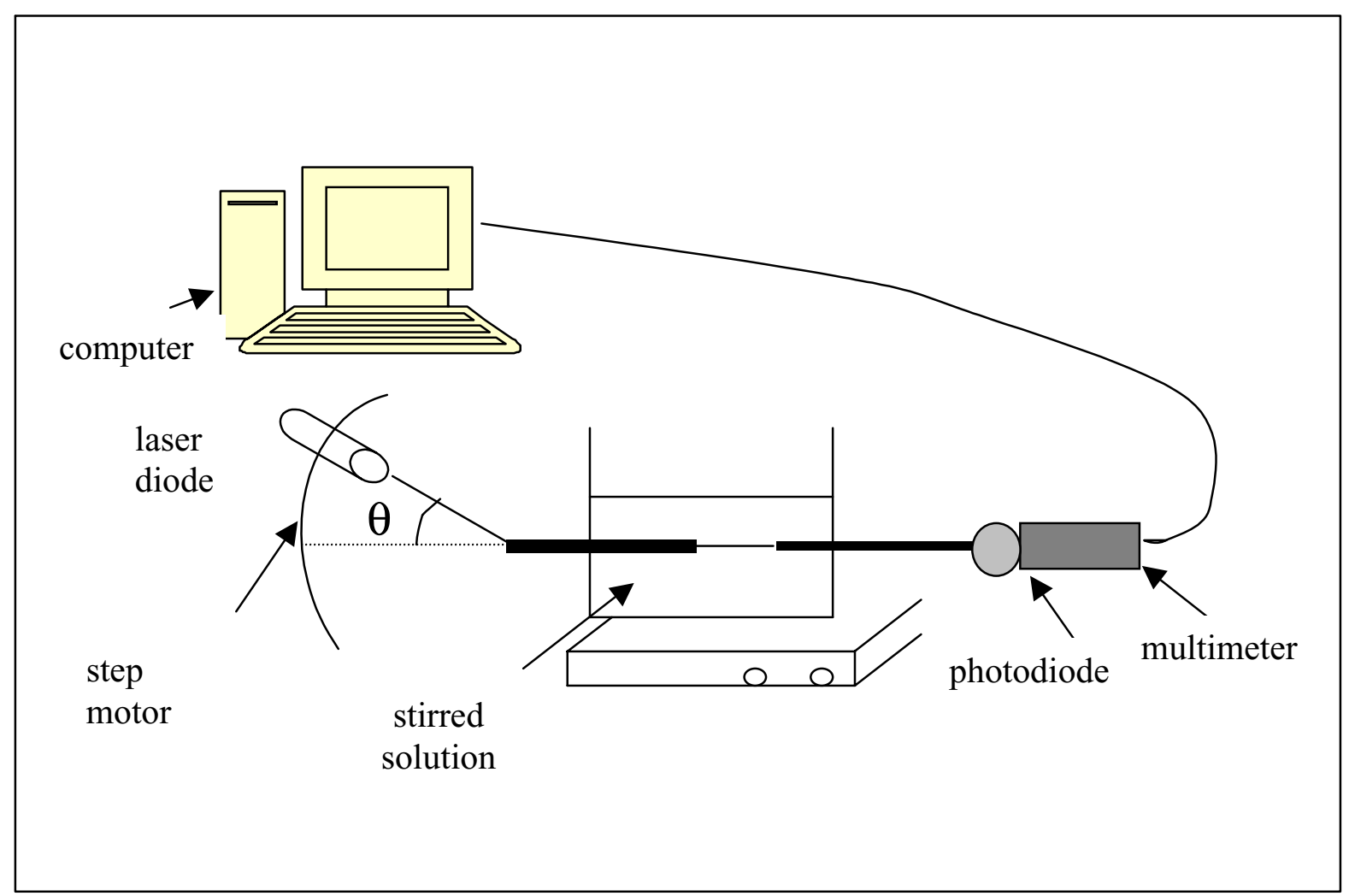

Figure 1. Experiment set-up.

This paper deals with the development of an optical fibre refractometer for aqueous medium and for toluene traces detection. We use a multimode optical fibre with $30 \mathrm{~cm}$ length. We study the calibration of the refractometer with different liquid mixtures. We use this refractometer to measure the refractive index of two volatile compounds such us fuel and unleaded gas. We utilise a sol-gel polymer 
(Tetrathoxysilane, TEOS) for chemical detection. The polymer can be deposited onto the fibre with a deep-coating method. The sensibility and the stability of the two aspects will be studied.

\section{Experimental}

\section{Experimental set-up}

The experimental set-up is shown in Figure 1. The light source was a laser diode with collimated beam which emitted in the visible range $(670 \mathrm{~nm})$, and which is injected into the optical fibre. This laser diode was attached on a rotation stage which connected is to step motor. The motor is connected to a DC alimentation and to a computer. The transmitted light in the input end of the fibre was totally collected on the $16 \mathrm{~mm}^{2}$ area of a silicon photodiode. The transmitted light is converted to a voltage through a simple electric model which contains an amplifier and a potentiometer. We read out the electric voltage by means of a highly sensitive multimeter which is connected to the computer. The computer is able to communicate with the step motor and the multimeter. The angle of incidence can be fixed with an accuracy of 0.1 degree.

For refractometer measurements, we use a mixture of triethylene-glycol $(n=1.453)$ and distilled water ( $\mathrm{n}=1.335)$ to make the calibration of the refractometer. During all experiments, the mixture is stirred to have a homogeneous and transparent medium.

\section{Preparation of the optical fibre}

The optical fibre was a multimode silica/silicone fibre (PCS 400, core diameter $400 \mu \mathrm{m}$ ). The refractive indices of the core and the cladding were 1.457 and 1.407 , respectively, leading to a numerical aperture of 0.37 . The fibre part was prepared in a two step procedure : (1) removing the silicone cladding on a length of $15 \mathrm{~mm}$ and (2) polishing the two extremities of the fibre. The total length of the fibre is about $30 \mathrm{~cm}$.

\section{Sol-Gel polymer}

A xerogel sensing layer was applied on the bare core by dipping the fiber into an input sol and withdrawing it with a velocity of $10 \mathrm{~cm} / \mathrm{min}$. The sols based on tetraethoxysilane (TEOS) were prepared from the alkoxides, ethanol, water and $\mathrm{HCl}$. The applied gel layer were converted into xerogel layers by drying at $90^{\circ} \mathrm{C}$ for 24 hours. The thickness of the layers were below $500 \mathrm{~nm}$. More details can be found in reference [8].

\section{Evanescent Wave Sensing: Background}

The sensor configuration employed by us relies on the evanescent wave (EW) interaction between the guided radiation and the analyte-sensitive reagent. The evanescent wave is the exponentiallydecaying electric field which penetrates a short distance into the low index medium, when the total internal reflection occurs at a dielectric interface. The degree of penetration is often characterised by 
the depth, $d_{p}$, which is the perpendicular distance from the interface at which the electric field magnitude, $\mathrm{E}$, has fallen to $1 / \mathrm{e}$ of its value, $\mathrm{E}_{0}$ at the interface

$$
\mathrm{E}=\mathrm{E}_{\mathrm{o}} \exp \left(\frac{-\mathrm{z}}{\mathrm{d}_{\mathrm{p}}}\right)
$$

The magnitude of the penetration depth is given by:

$$
\mathrm{d}_{\mathrm{p}}=\frac{\lambda}{2 \pi \mathrm{n}_{1}\left[\sin ^{2} \theta-\left(\frac{\mathrm{n}_{2}}{\mathrm{n}_{1}}\right)^{2}\right]^{\frac{1}{2}}}
$$

Where $\mathrm{z}$ is a position in the $\mathrm{Z}$ axis, $\lambda$ is the vacuum wavelength, $\theta$ is the angle of incidence to the normal at the interface, and $\mathrm{n}_{1}, \mathrm{n}_{2}$ are the refractive index values of the dense and rare media, respectively.

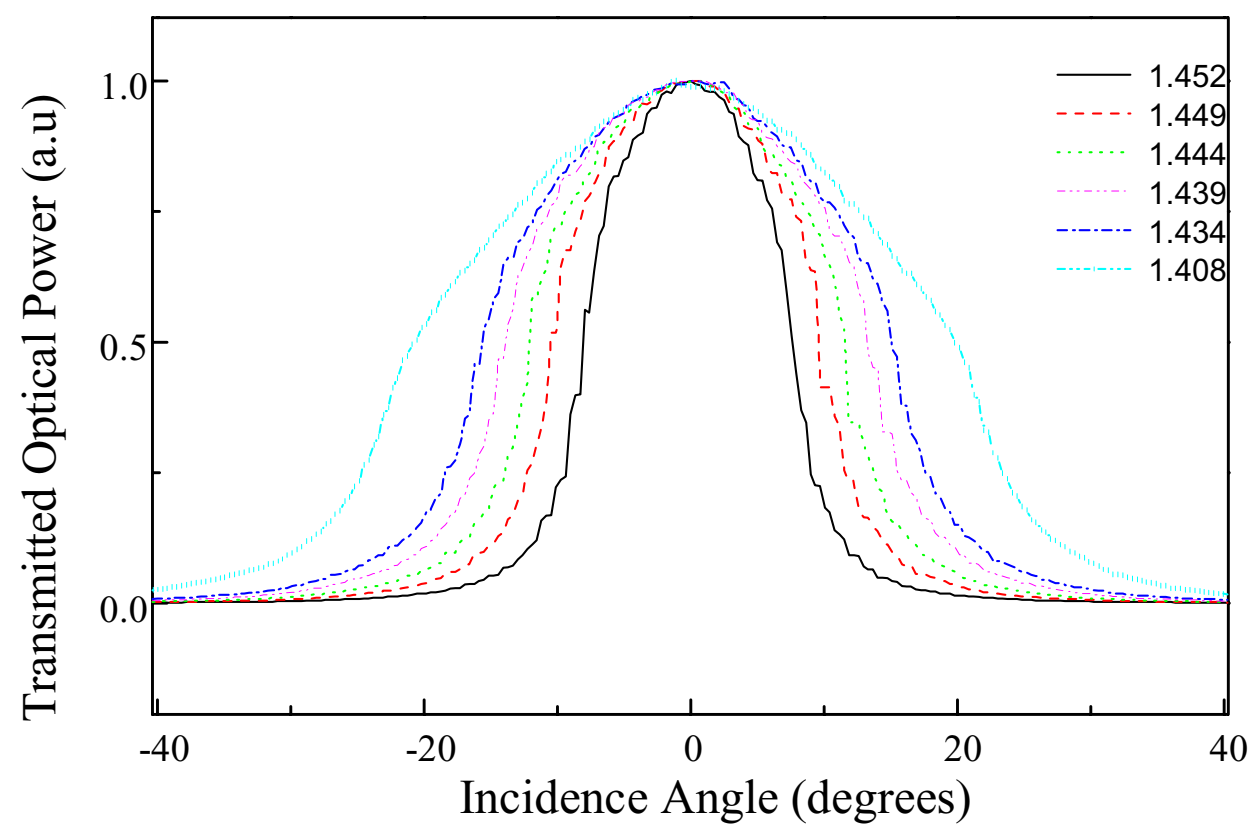

Figure 2. Experimental curves of the light power transmitted versus the incidence angle $\alpha$ for different refractive indices.

\section{Results and Discussions}

\section{Refractive index calibration}

First of all, the entrance end of the optical fibre must be perfectly cleaved and adjusted on the axis of rotation of the step motor. We moved the entrance with a micrometric translation stage to obtain the maximum transmitted light power. Then, we carried out a calibration curves with maximum values of 
refractive index. Figure 2 shows experimental curves in different water-triethylene glycol mixtures of the normalized optical power transmitted versus the angle of incidence for an optical fibre with $15 \mathrm{~mm}$ non-cladded length. Assuming that all the rays carry the same light power and neglecting light scattering at the core-cladding interface, the light power transmitted through the fibre remains constant for $\alpha<\alpha_{\mathrm{NA}}$ and decreases sharply if $\alpha>\alpha_{\mathrm{NA}}$, where $\alpha_{\mathrm{NA}}$ is the numerical aperture of the fibre. Furthermore, we can see that for a fixed angle (for example 10 degrees) the sensitivity of the refractometer increase when the refractive index of the medium is nearly the refractive index of the core.

Figure 3 shows the theoretical curves of variation of the optical power versus the angle of incidence in the case we take account diffusion phenomena. This calculations can be done with a simple Fortran program after the Maxwell equation resolution. The interface between the core and the cladding can be considered as a plane surface because the fibre is a multimode fibre with $600 \mu \mathrm{m}$ core diameter, which is superior to the wavelength used. To calculate the optical power at the end of the fibre, we have to calculate the reflection coefficient of each rays insight the fibre. Such a calculation can be done through Maxwell Law's.

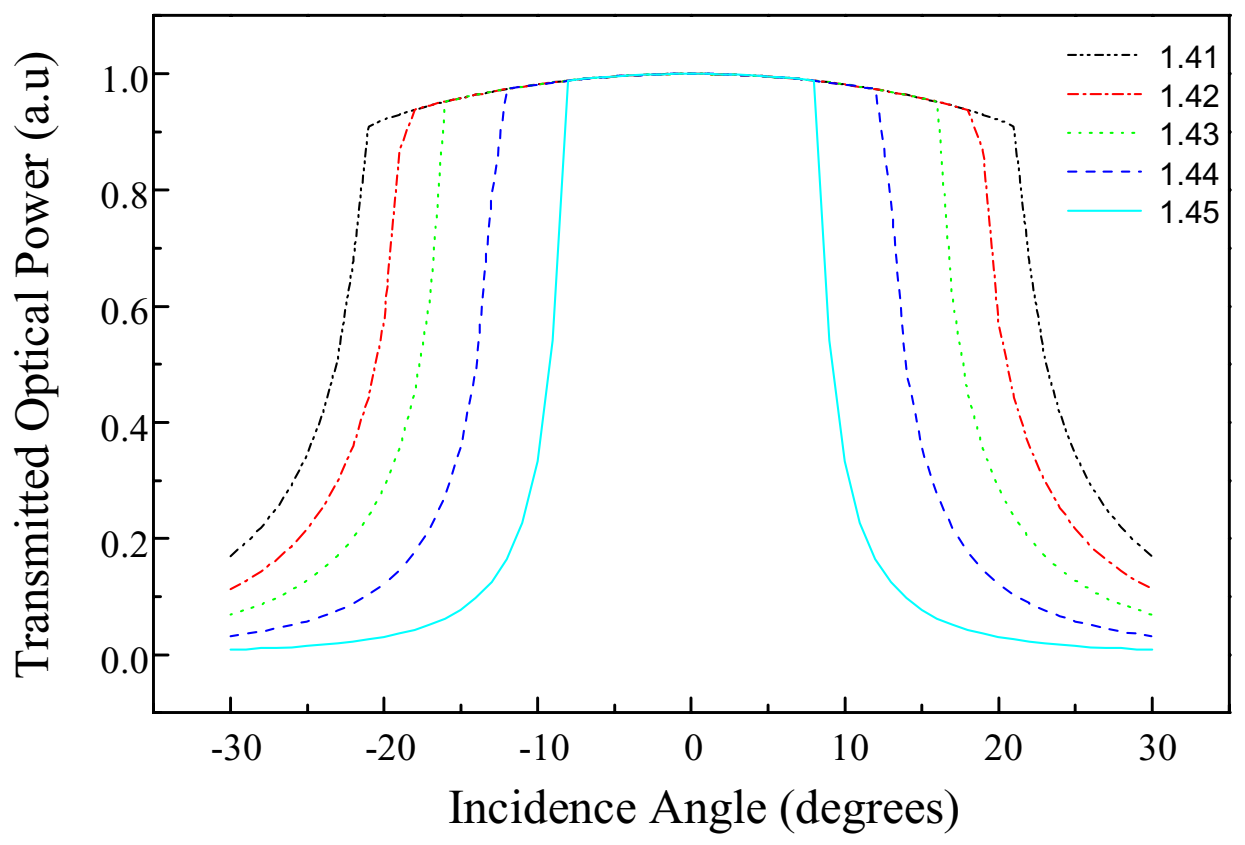

Figure 3. Theoretical curves of the optical power transmitted versus the angle of incidence $\alpha$ for different refractive indices.

We also studied, as presented in Figure 4, the variation of the normalised optical power versus the refractive index of the mixture for different angles of incidence. The slope of these curves gives an idea about the sensitivity of the refractometer. As you can see, the curves exhibit different slopes, which depend on the refractive index region. Each curve is fitted by linear branches whose slopes are related to the sensitivity of the sensor: the steeper the slope, the better the sensitivity. 


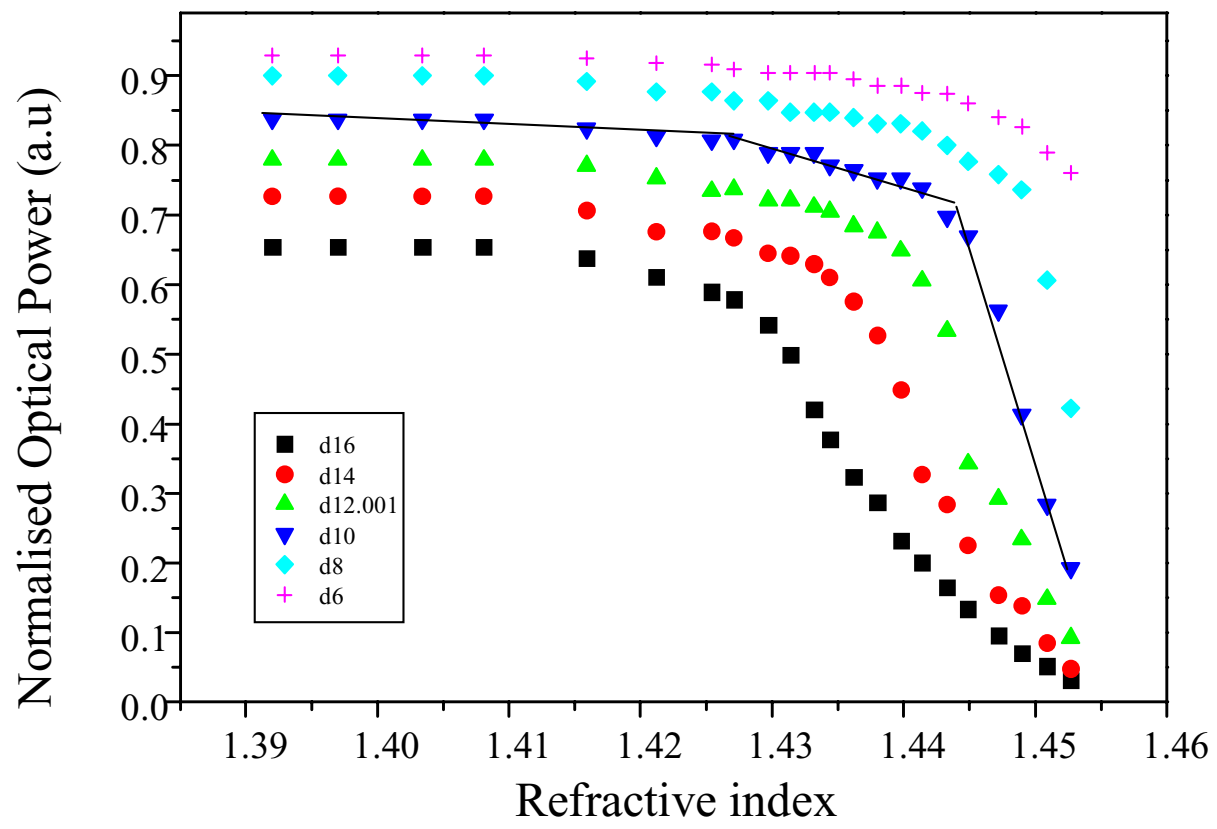

Figure 4. Experimental response of the refractometer to variation of the dielectric refractive index for several angles of incidence $\alpha$.

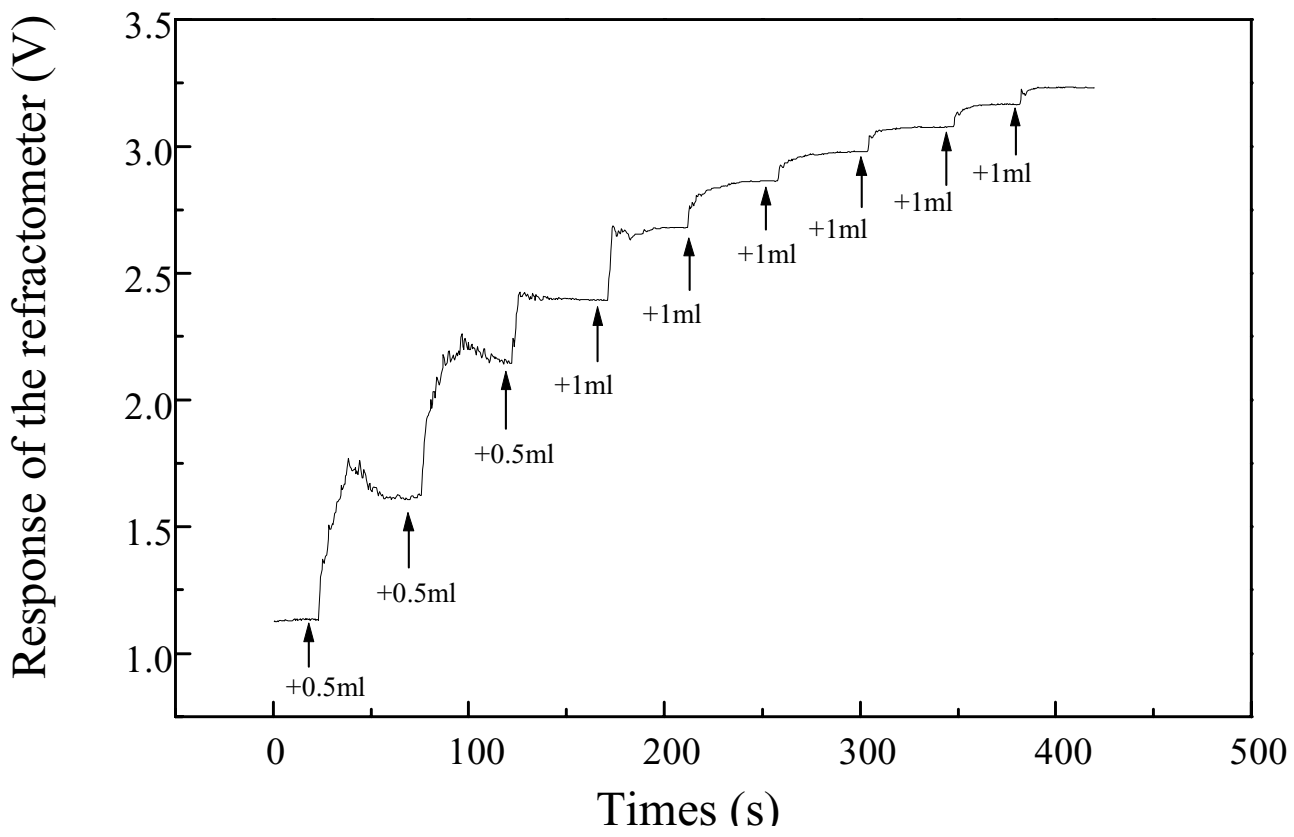

Figure 5. Experimental response of the refractometer to the variation of water volume for fixed angles of incidence $\alpha$ (10 degrees). The sensitivity decreases when the refractive index is far from the refractive index of the core. 


\section{Time dependence}

We studied the variation of the normalised optical power for a fixed angle of incidence (10 degrees) versus the time, when we add distilled water in triethyleneglycol (Figure 5). One can recognise that the sensitivity decreases when we increase the volume of distilled water. This is due the decrease of refractive index of the mixture (from 1.457 to 1.407), which justifies the results in Figure 4 . The response of the refractometer is about 2 seconds.

\section{Refractive index measurement}

We have tried to measure the refractive index of two volatile compounds: unleaded gas and fuel. As both compounds are volatile at room temperature and absorbent, it was impossible to measure their refractive indices with an Abbe Refractometer. Therefore, our optical fibre refractometer was used.

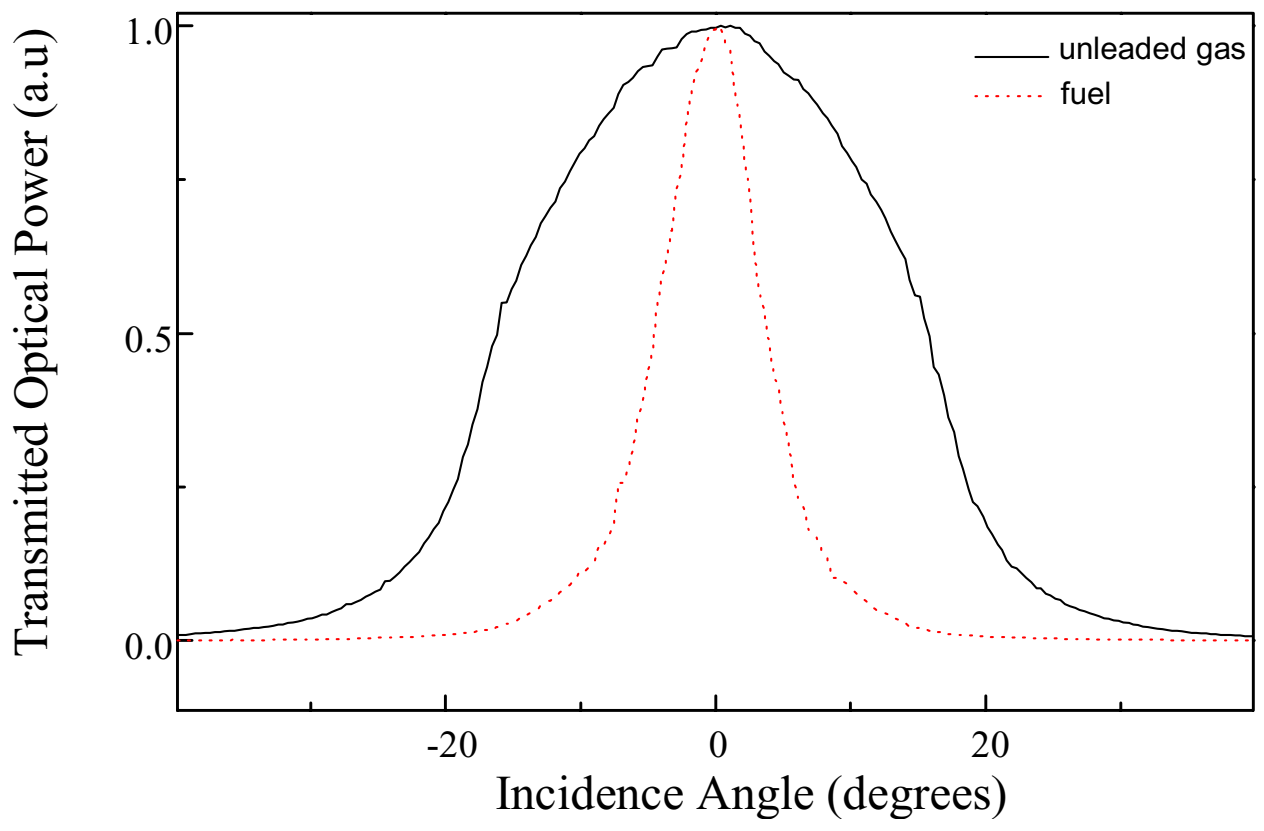

Figure 6. Experimental curves of the light power transmitted versus the incidence angle $\alpha$ for unleaded gas and fuel.

Figure 6 shows the normalised optical power versus the angle of incidence in the case of both compounds. We can easily distinguish between these two compounds with our optical refractometer, which can be applied for controls in distribution or in transportation of fuels.

The superposition of these curves with calibration curves or with theoretical curves (Figure 2) allows to determine the refractive index of the fuel and unleaded gas at room temperature: $\mathrm{n}_{\text {fuel }}>1.45$ and $\mathrm{n}_{\text {unleaded gas }}=1.434$. 


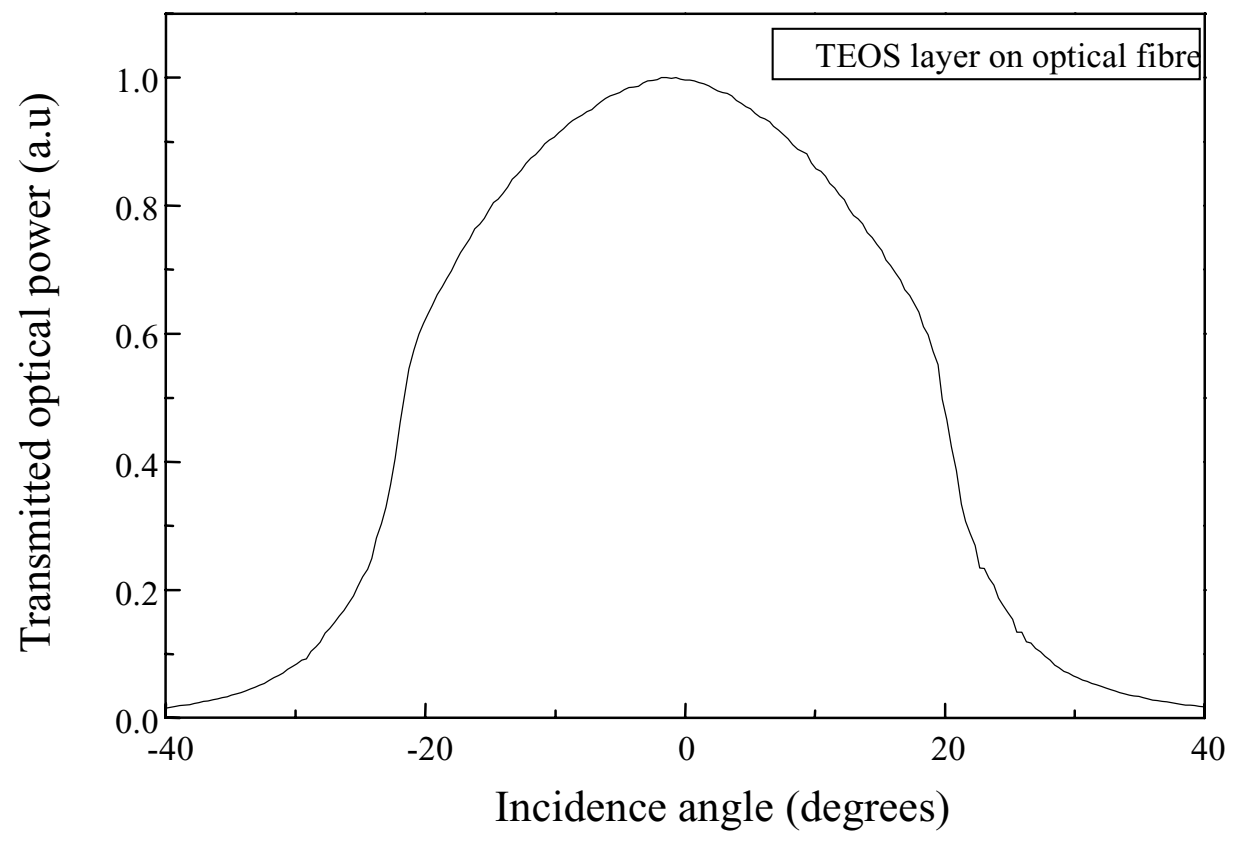

Figure 7a. Experimental curves of the light power transmitted versus the incidence angle $\alpha$ for an optical fibre coated with xerogel.

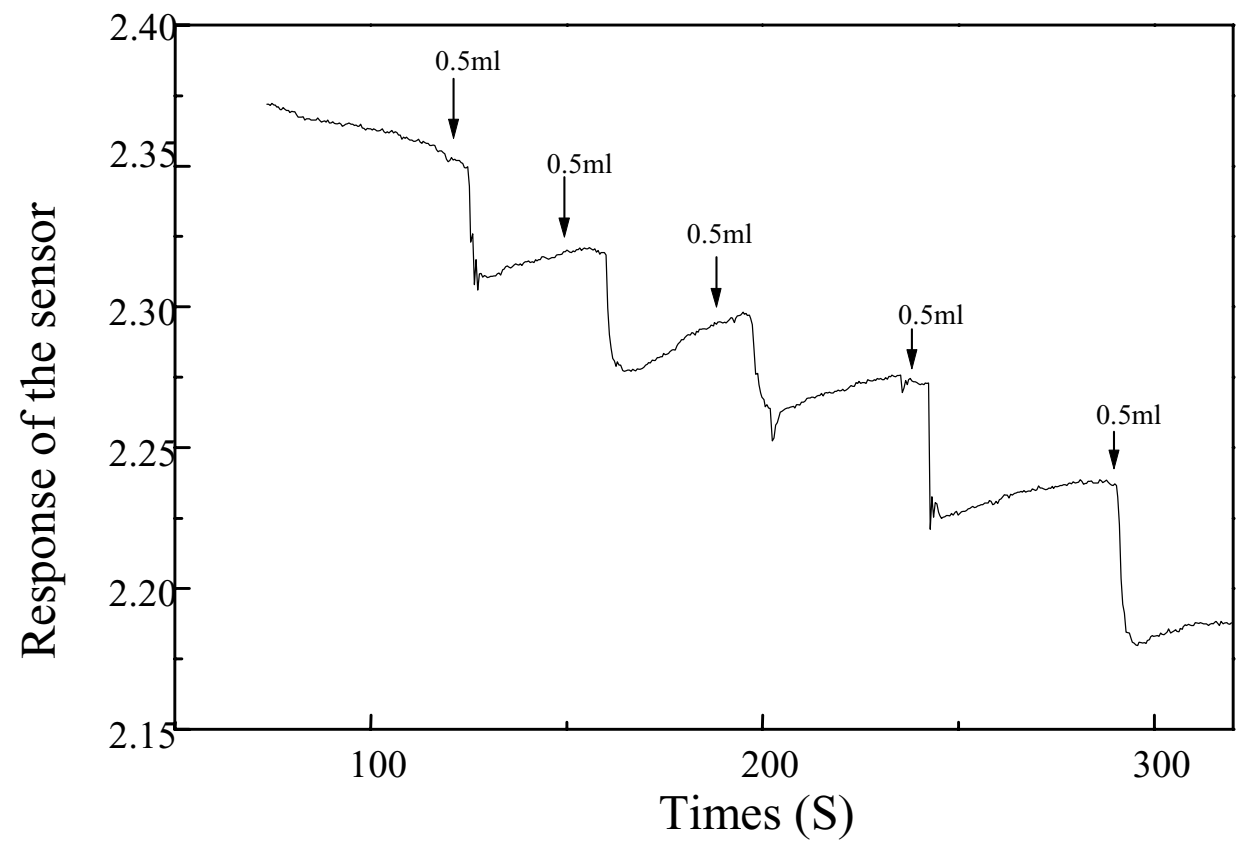

Figure $7 \mathbf{b}$. The response of the sensor coated with xerogel for toluene in water for fixed angle. 


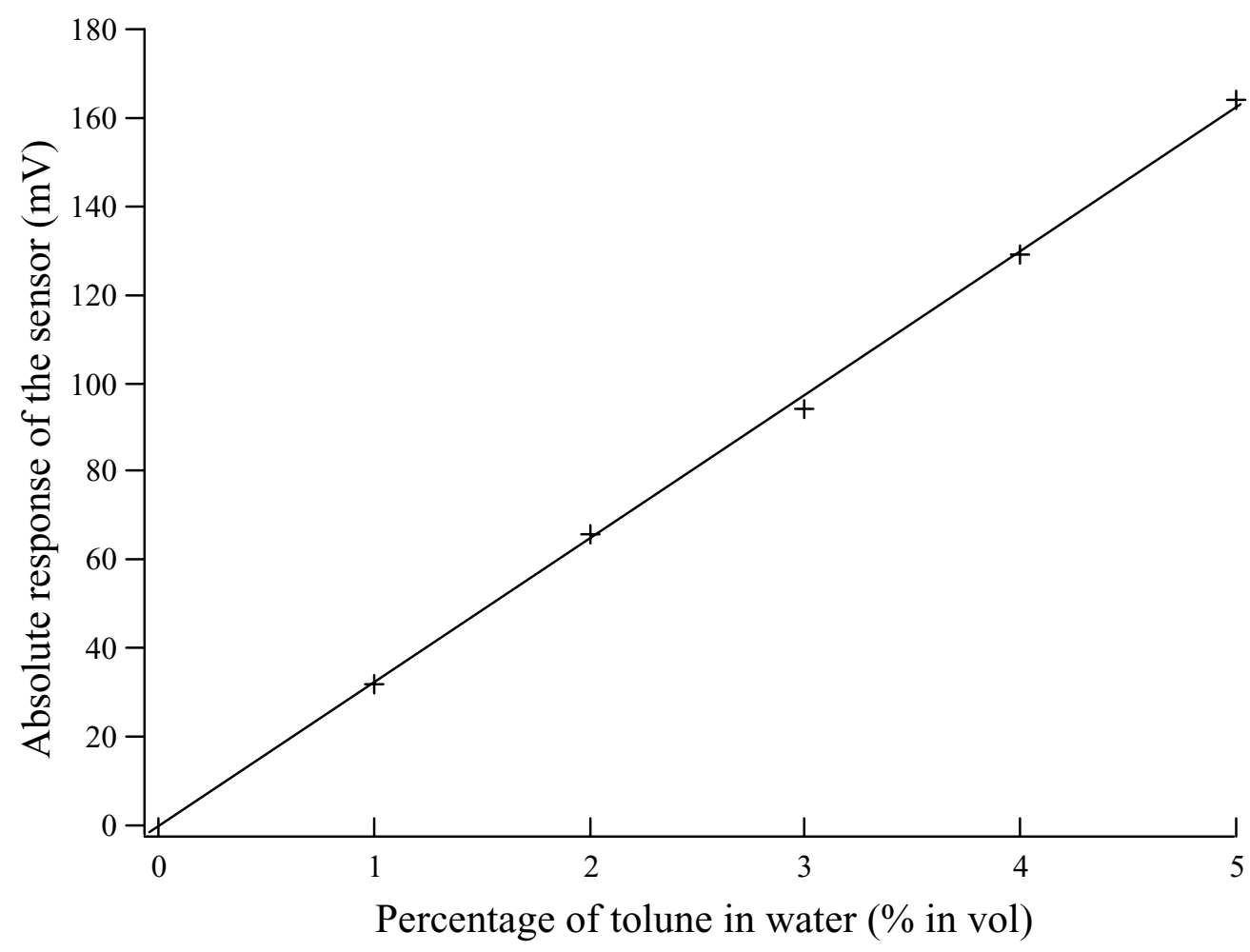

Figure 7c. The absolute response of the sensor to different percentage of toluene in water.

\section{Chemical detection}

Figure 7a shows experimental curves of the light power transmitted versus the incidence angle $\alpha$ for an optical fibre coated with xerogel in water. The response of the sensor coated with the xerogel for different injected volumes of toluene in water is shown in figure $7 \mathrm{~b}$. The total volume of water is 50 $\mathrm{ml}$. This response has been obtained with an incidence angle of 22 degrees ( 22 degrees is equal to $\alpha_{50}$ which is the angle for which the transmitted light power is half of its maximal value) [9]. A decrease of the transmitted light power is observed which indicates an increase in the refractive index of the xerogel in presence of toluene due to partition of toluene between xerogel and water. Figure 7c shows the absolute response of the sensor to different percentages of toluene in water. The response is linear and leads to a detection limit about $1 \%$ which correspond to $32 \mathrm{mV}$. We estimate to detect a lower percentage if we dope our sol-gel layer.

\section{Conclusions}

We developed an optical fibre sensor suited for aqueous medium that is based on the excitation of an evanescent wave at the core/cladding interface. Refractive indices of absorbent and volatile compounds such as fuel and unleaded gas were determined. Using a xerogel sensing layer as optical cladding, a quantitative toluene detection in water was performed. For future work, we will use other sol-gel layers for ions detection. The stability and the reproducibility of the sensor will be investigated. 


\section{Acknowledgements}

The authors thank the Alexander Von Humboldt Foundation for the material donation.

\section{References}

1. Abdelghani, A.; Chovelon, J. M.; Jaffrezic-Renault, N.; Ronot-Trioli, C.; Veillas, C.; Gagnaire, H. Sensors and Actuators B 1997, 38-39, 407-410.

2. Abdelghani, A.; Chovelon, J. M.; Jaffrezic-Renault, N.; Veillas, C.; Gagnaire, H.; Analytica Chimica Acta 1997, 337, 225-232.

3. Jorgenson, R. C.; Yee, R. C.; Karlsen, S. R. Proceeding of the Transducers93 Meeting, Yokoham, Japan, June 1993.

4. Ronot-Trioli, C.; Trouillet, A.; Veillas, C. Sensors and Actuators A 1996, 54, 589-593.

5. Mac Craith, B. D.; McDonach, C.; Butler, T. Journal of Sol-Gel Science and Technology 1994, 2, 661-665.

6. Abdelghani, A.; Chovelon, J. M.; Jaffrezic-Renault, N.; Lacroix, M.; Gagnaire, H.; Veillas, C.; Berkova, B.; Chomat, M.; Matejec, V. Sensors and Actuators B 1997, 44 (1-3), 495-498.

7. Berkova, D.; Sedlar, M.; Matejec, V.; Kasik, I.; Chomat, M. Abdelghani, A.; Jaffrezic-Renault, N.; Lacroix, M. Journal of Sol-Gel Science and technology 1998, 13, 569-573.

8. Burck, J.; Conzen, J.P.; Beckhaus, B.; Ache, H. J. Sensors and Actuators B 1994, 18-19, 291.

9. Ronot, C.; Archenault, M.; Gagnaire, H.; Goure, J.P. Sensors and Actuators B 1993, 11, 375-381.

Sample Availability: Available from the authors.

(C) 2002 by MDPI (http://www.mdpi.net). Reproduction is permitted for noncommercial purposes. 